\title{
CORRECTIONS
}

\section{Early fluid resuscitation in severe trauma}

The authors of this Clinical Review (BMJ 2012;345:e5752, doi:10.1136/bmj.e5752) would like to point out that there are a few inaccuracies in their article. In the last line of the first paragraph, the parenthesis should have clarified that severe traumatic injuries are defined as $>15$ [not " $<15$ "] by the injury severity score. The third paragraph under the heading "What fluids should be used to resuscitate trauma patients who do not need DCR?" should also have read, in its penultimate sentence: "Among patients with severe traumatic brain injury not in hypovolaemic shock, initial resuscitation with either hypertonic saline or hypertonic saline and dextran [not "or dextran"], compared with normal saline, did not result in improved neurological outcome or survival at six months." Lastly, reference 16 should have cited Bickell WH [not "Bicknell"] as the first author.

Cite this as: BMJ 2012;345:e6248

๑) BMJ Publishing Group Ltd 2012 